\title{
Trends in Software Design for Distributed Computing Systems
}

\author{
Stephen S. Yau, Xiaoping Jia and Doo-Hwan Bae \\ Software Engineering Research Center and \\ Computer and Information Sciences Department \\ University of Florida \\ Gainesville, Florida 32611 USA
}

\begin{abstract}
Due to the rapid development of VLSI technology, computing systems can be made much more distributed and to match the structures of the problems for various applications. Because the characteristics of distributed computing systems are significantly different from those of centralized computing systems, different approaches are needed to effectively address the design issues related to the characteristics of software for distributed computing systems. In this paper, the methods currently used to develop the software for distributed computing systems are classified into three categories: dataflow-oriented, communicationoriented, and object-oriented. Their applicabilities are compared, and the future trends of software design approaches are discussed. Object-oriented software design methodology is identified as a more promising approach to software design for distributed computing systems.
\end{abstract}

Index Terms- Distributed software design approaches, distributed computing systems, dataflow-oriented design approach, communication-oriented design approach, object oriented design approach, future trends.

\section{Introduction}

Due to the rapid developrrent of VLSI technology, computing systems can be made much more distributed and to match the structures of the problems for various applications. Furthermore, distributed computing systems have the following major advantages: 1) The performance of a distributed computing systen can be greatly improved by increasing its parallel processing capability. 2) A distributed computing system can be made more fault tolerant due to its distributed nature. 3) Thr: system can be easily expanded. Because the characteristics of distributed computing systems are significantly different $f$.om those of centralized computing systems, we need differ $2 n t$ approaches to software design which can effectively address the issues related to the characteristics of distributed cr mputing systems, and realize the full potential of distributer computing systems.

In this paper, we will c onsider the characteristics of distributed computing syster $;$ and categorize the methods currently used to develop soitware for distributed computing systems in three classes: $d$ ttaflow-oriented, communication- oriented, and object-oriented. The problems and issues to be addressed by the software design methodologies for distributed computing systems will also be identified. We will also discuss the trends of software design methods and identify the object-oriented software design methodology as a more promising approach $\%$ software design for distributed computing systems although many research problems remain open.

\section{Characteristics of Distributed Computing Software Systems}

A distributed computing system can be considered as a number of hardware processors with each processor having certain computing capability, and a number of communication links among processors. From software point of view, a distributed software system an be considered as a set of sequential processes with earh process residing on a hardware processor, and the processes cooperate with one another to fulfill a system-wide task. A.lthough the development of these individual processes exhibi $s$ many of the common characteristics shared by the development of sequential software for centralized computing systems, the following characteristics of the software for distributed computing systems are distinct and must be addressed by any software design methodology for such systems:

- The processes in a d.stributed software system can be executed in parallel.

- The processes in a distributed software system are able to cooperate with one another to fulfill a system-wide task. The cooperation involves communication and synchronization among the processes.

- Software components can be mapped into hardware resources by partitioni: $g$ and allocating software components based on the st cucture and performance requirements of the distributed computing system.

Traditionally, the para! elism and the cooperation among the processes of a distribut $\approx$ software system are supported either by the underlying (distributed) operating system or by the programming langı age, in which the software is implemented. In the former method, the parallelism and the cooperation among the processes are achieved by invoking the utilities provided by th : operating system from conventional sequential programn ing languages. V-kernel [1], Mach 
[2] and LOCUS [3] are t\} ree of the distributed operating systems that provide suck utilities. In the latter method, the parallelism and the cocperation among the processes are supported by programming, language constructs provided by concurrent programming languages, such as Ada [4], Concurrent C [5] and Occam [5].

While such kinds of support are suitable for simple applications, this approach becomes insufficient for large-scale and complex applications because the communication and synchronization have to be handled at the implementation level, and the modularity and modifiability of the software system are reduced. It also hinders portability of the software system because its implementation depends on the underlying programming language, operating system and architecture. Thus, it is necessary for the design methodologies for distributed software systems to support the exploitation and maximization of parallelism, the cooperation among the processes, and the mapping of software components into available hardware resources.

\section{Software Design Methods for Distributed Computing Systems}

Current software design methods for distributed computing systems can be characterized as dataflow-oriented, communication-oriented, and object-oriented based on their emphases on different aspects of the distributed software systems and the ways to decompose software systems. We will discuss each of these categories in more detail.

\section{Dataflow-Oriented Design Methods}

In dataflow-oriented design methods, a distributed software system is decompose 1 into modules according to their functionalities. Each module is considered as a sequential process. Because communication and synchronization are not incorporated in he design phase but supported by concurrent programmitig languages in the implementation phase [7], the dataflow-oriented approach to the software design of distributed computing systems is the same as that of centralized computing systems. Structured Analysis(SA)/Structured Design(SD) $[8,9]$ is a classic datafloworiented design method. In the Structured Analysis, a software system is represtnted by functions, dataflows between functions, and data stores using dataflow diagrams. Functions are computations that the system carries out. Dataflows show the relations between functions. Data stores are the collections of data used throughout the system execution. Functions in dátaflow diagrams can be decomposed hierarchically. Datafow diagrams are used with data dictionaries and process srecifications [10]. In conjunction with Structured Analysis, "itructured Design is often used in dataflow-oriented design methods to obtain well-structured design of software systems Structured Design uses two criteria to evaluate the quality of a design: coupling and cohesion of modules. The objective of Structured Design is to design a system in which modules have high cohesion and low coupling. This approz is influenced by the nature of the subprogram (or proceuture) and thus is suitable for rep- resentation of algorithmic abstraction.

The design of distributed software systems using SA/SD involves the following major steps.

1) Identify the external entities, data stores and functions in the system and specify the data dependency among them using dataflow diagrams.

2) Transform the dataflow diagrams into the design of the software system in the strecture charts.

3) Implement the software system based on the structure charts.

Although this method has been used extensively and supported by many tools, it hes the following drawbacks when applied to the design of distributed software systems:

- absence of data abstraction and information hiding mechanisms.

- inadequacy for applications with natural concurrency.

- inadequacy for suppcrting communication and synchronization.

- irreponsiveness to changes in the problem space.

Therefore, this approach is only appropriate for applications in which each process involves intensive computation while the communication among the processes is rare. There are also several other dataflow-oriented design methods, which are variations of SA/SD, such as SADT $[11,12]$ and SA/RT [13]. SADT uses the box-and-arrow diagram technique in which a box represents data or activity, and directed links connected to left, up, right, and down sides of the box represent input, control, output, and mechanism, respectively. In SA/RT, the dataflow diagram is augmented by controloriented features in order $t a$ address problems in distributed real-time designs.

An example of a distributed software design using SA/SD is shown in Figures 1 and 2. The system being developed is a hypothetical system that monitors its environment by a sensor, displays the status of its environment via a display device and controls its environment via a control device. In spite of the simple nature of this example, it captures the essence of a variety of applications such as auto-pilot systems, cruise-control systems, and process control systems. Figure 1 shows the dataflow diagram of the distributed software system, in which functions are represented by rounded rectangles, dataflows are represented by directed arcs, data stores are represented by parallel bars, and external devices are represented by rectangles with shadows. This system consists of three external devices: Sensor, Display_Device, and Control_Device, three functions: Monitor_Process, Display_Process, and Control_Process, and one data store: Buffer. Input data from Sensor is transformed by function Monitor_Process, and stored in data store Buffer. Then, data in Buffer is fetched by Displa ${ }_{-}$Process and Control_Process. In Structured Design [9], the structure chart is used to represent modules which will be implemented as functions or procedures. Figure 2 shows the structure chart for this software system. The software syst $\mathrm{m}$ is driven by a main module on 


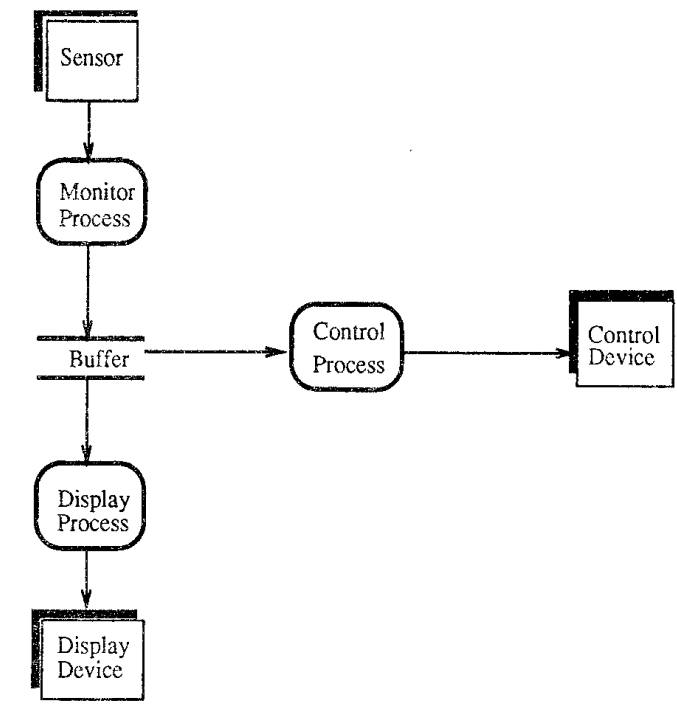

Figure 1: The dataflow diagram of a monitor-control system.

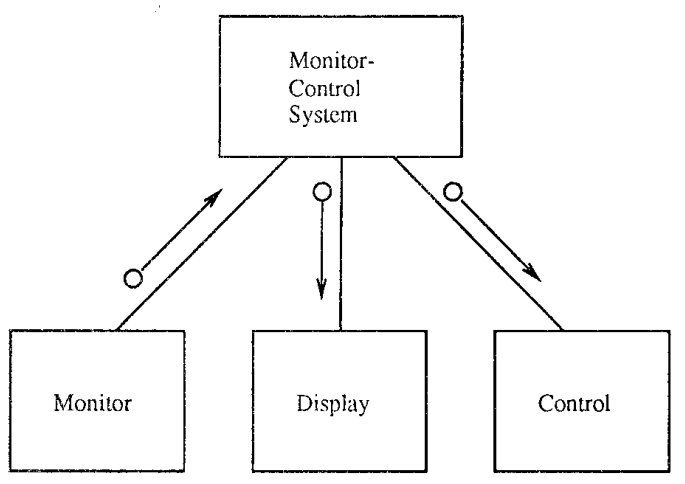

Figure 2: The structure ch.rt of the monitor-control system shown in Figure 1.

the top of the structure chart. Three submodules represent functions identified in the dataflow diagram. Figures 1 and 2 show the high level design of the software system. In the detail design of the system, the functions and data types need to be defined. For example, the details of the transformation of the Monitor-Process that transforms the raw data input from the Sensor to the data stored in the Buffer, which will be fetched by the Control_Process and Display_Process, need to be defined, as well as the data structures of the Buffer.

\section{Communication-Oriented Design Methods}

In communication-orientec design methods, a software system is decomposed into a set of processes or tasks. The behavior of each process is specified in terms of communication and synchronization among the processes. In contrast to dataflow-oriented design methods, communication-oriented putation and data structures are relatively simple, while the handling of various communication and synchronization is the dominant issue. Such applications include inherently distributed systems and communication intensive applications. SREM [14], STATEMATE [15], Raddle [16] and various Petri-net based methods $[17,18,19]$ are examples in this category.

Among these methods, those based on the Petri net are probably the most well-known and most well-defined methods. A Petri net consists $c^{2}$ two disjoint sets of nodes, called places and transitions, anc a number of tokens. The places and transitions are relater with directed links representing the flow of control or data. Places are represented by circles, transitions are representec by straight bars, and tokens are represented by small dots in places. Initially, a number of tokens are located in some places. Changes in the Petri net occur when one or more tzansitions are fired. A transition can be fired when every place immediately preceding this transition has at least one token. Once a transition is fired, a token from each of those preceding places is removed, and a token is added to the places immediately following that transition. In this way, the dynamic behavior of concurrent processes can be specified in terms of communication and synchronization among processes.

There are three communication modes among the processes: asynchronous, syncironous or remote invocation. In the asynchronous communication mode, the sender sends a message and proceeds without waiting for an acknowledgment from the receiver. In the synchronous communication mode, the sender sends a message and waits for an acknowledgment. This mode limits potential concurrency of distributed software systems because the sender may spend a substantial amount of unnecessary time waiting for the acknowledgment from the receiver. The third communication mode is remote invocation. In this case, the sender waits for the results of computation from the receiver. There are specific patterns for each of these different communication modes expressed in the Petri net [20].

The software design for distributed computing systems using Petri nets involves the following steps:

1) Identify concurrent processes in the problem domain.

2) Identify the communication channels among these processes and the communication modes, which can be synchronous, asynchronous, or remote invocation.

3) Define the computation and data structures associated with the Petri net.

4) Translate the Petri-net representation into implementation. Automated tools are available to translate Petri-net representation into implementation in Ada [21] or OPS5 [22].

The design of a distributed software system using the Petri-net approach is shown in Figure 3. This is the same example used to illustrate tne dataflow-oriented design methods discussed before. The three concurrent processes, Monitor_Process, Display_Process and Control_Process are identified by dotted rectangles. The communication among these 


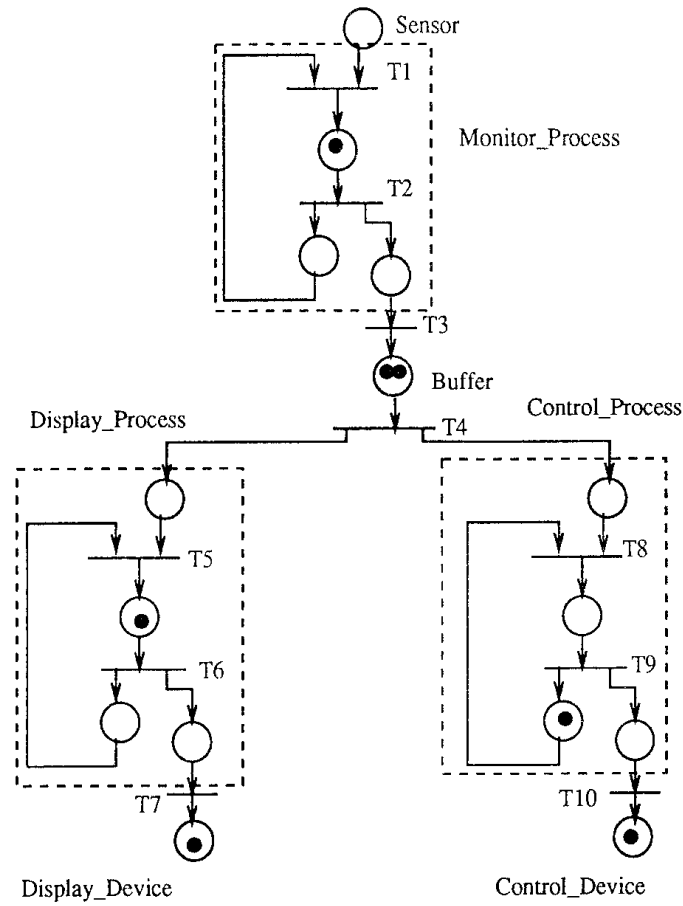

Figure 3: A Petri-net ref resentation of a monitor-control system.

processes is asynchronous via a buffer. Monitor_Process is initiated by firing the transition at the external device place. At the transition T2, tokens are located at its two following places. One place is for controlling the transition $\mathrm{T} 1$, and the other is for storing data into a buffer. Display_Process and Control_Process are activated at the same time by firing transition T4. Both control flow and data flow can be represented in the Petri net. The path of a token, T1 $\rightarrow$ $\mathrm{T} 2 \rightarrow \mathrm{T} 1$, represents cont:ol flow, and the path of a token, $\mathrm{T} 1 \rightarrow \mathrm{T} 2 \rightarrow \mathrm{T} 3$, represents data flow. This capability of representing both control flow and data flow in one graph is considered as a major ar vantage of using Petri-net representation [17], but the distinction between these two flows is implicit. Thus, the comprehensibility is hampered. After specifying communication aspects of the software system using the Petri net, the functions and data structures need to be specified. In our example, the function that transforms the raw data input from the sensor to the data stored in the buffer needs to be defined and attached to the transition T2. It will be invoked when the transition $\mathrm{T} 2$ is fired. The functions for displaying and controlling also need to be defined, and attach to transitions $: 6$ and $T 9$, respectively.

\section{Object-Oriented I'esign Methods}

In object-oriented design methods $[23,24]$, a software system is decomposed into a set of cooperating objects. Each object corresponds to an entity such as data, action, or hardware device. The basis of object-oriented design methods is the concept of classes and objects. A class denotes a set of objects sharing some common properties. An object is realized as a unique instance of a class. An object is a logical entity that encapsulates some local data and operations, called methods. The local data within an object is hidden from other objects. Interaction between objects can only occur via message passing which is the communication mechanism used in object-oriented methods. An object can send a message to another object. Upon receiving the message, the receiver decides whether $i_{s}^{t}$ executes one of its methods by accessing and possibly updating its local data. The objectoriented design approach is suitable for software design for distributed computing systems because the concept of classes and objects and the organization of objects naturally reflect the structure of the problem domain of many distributed computing applications, and objects provide natural units for parallelism.

The software design of distributed computing systems using object-oriented design approach involves the following major steps [23]:

1) Identify objects and their attributes. All physical or logical entities in the problem domain are recognized. New classes can be defined by inheriting the local data and methods of their superclasses.

2) Identify the methods frovided by and required of each object. The static semantics of the object is established by determining the methods. The dynamic behavior of each object is established by identifying the constraints upon the time or space.

3) Establish the visibility of each object in relation to other objects. The static dependencies among objects and classes of objects are identified.

4) Establish the interface of each object. This captures the static semantics of each ofject or class of objects.

5) Implement each object by choosing a suitable representation for each object or class of objects and implementing the interface.

An example of object-oriented design of a distributed software system is shown in Figure 4. This is the same example used to illustrate the dataflow-oriented and communication-oriented design methods. First, four different objects are identified: Sensor, Buffer, Control_Device, and Display_Device. Their dependency relations based on the massage paths are represented by directed links. Figure 4 shows the decomposition and the structure of the software system. The differences between the object-oriented designs and the previous two categories of design methods are evident. In contrast to the two previous methods, the functional behavior of each object is not exposed to the outside world. Instead, it is embedded into objects as their methods. For example, function Display_Process in the dataflow diagram shown in Figure 1 is encapsulated into object Display.Device as a method. The method is invoked by receiving a message from another object Buffer. This information hiding pro- 


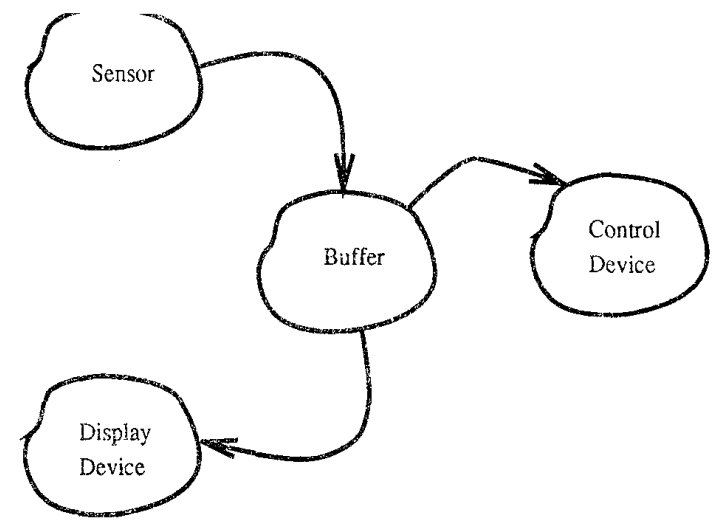

Figure 4: Object-oriented decomposition of a monitorcontrol system.

vides the protection of an object from illegal accesses, and aiso improves modifiability of the software systems. Secondly, the methods provided by each object need to be defined. For example, obje $\cdot t$ Buffer provides two methods: get-data and put-data. put.data is a method invoked by object Sensor to put data in the Buffer, get-data is a method invoked by objects Contro Device and Display_Device to retrieve data from the Buffe: The local data of object Buffer and the constraints, such as the size of the buffer, are also specified. Thirdly, the visibility of each object and its interface are established. The method put-data is only known to object Sensor, and the method get-data is only known to objects Display_Device and Control_Device. Sensor can only communicate with Buffer by invoking the method put-data in Buffer, and Control_Device and Display_Device can communicate with Buffer by invoking the method get-data. Finally, the objects defined in previous steps are implemented.

\section{Future Trends}

We have characterized methods for software design for distributed computing systems into three categories: datafloworiented, communication-oriented, and object-oriented. Dataflow-oriented design methods emphasize functional decomposition, whereas decisions on concurrency related issues are postponed to the implementation phase. Since the concurrency related issues, such as interprocess communication and synchronization, may directly affect the performance of the distributed software systems, the postponement of making decisions on concurrency related issues may result in poor performance and cause to redesign part of or even the entire software system in order to meet the performance requirement.

On the contrary, communication-oriented design methods deal with communication aspects of software systems as a primary task. Thus, concurrency related issues are naturally considered at the early stage of the design phase, and functional aspects of the distributed software system are handled as secondary concern. Furthermore, due to the lack of control and data abstraction mechanisms in this design ap- proacn, its applicauion is essentially restricted to communjcation intensive applications with relatively simple computation and data structures. One way to extend the capability of this design approach is to use the Petri net and to develop internal data structures to organize all control, data and auxiliary information within the Petri net. In order to take advantage of these two methods, a design method [25] has been proposed in which issues on both functional decomposition and comminication aspects are addressed at the initial stage of the design phase, and the validation of the design and distribution of processes into hardware processors can also be accomplished.

In object-oriented design methods, the software structure obtained reflects the structure of the application problem, and the data abstraction capability of objects supports information hiding. Thus, the comprehensibility, modularity, and modifiability of the software systems are enhanced. For these reasons, object-oriented design approach is more promising than the other two approaches to software design for distributed computing systems. However, the following problems in object-oriented design approach need to be solved in order to realize the full advantages of the approach:

- synchronization and parallelism support.

- integration of inheritance and parallelism.

- the development. of software tools to support objectoriented decomposition.

In the inherently distributed applications, such as the electronic-mail system on a network of workstations, simple message passing mechanism of object-oriented designs is not sufficient. Some synchronization primitives, such as semaphores and monitors, are needed to support the required parallelism. However, the adoption of these primitives will complicate the conceptual model of object-oriented design and affect the effectiveness of object-oriented design. Thus, more high-level and abstract mechanisms to handle these problems need to be develcped. Some object-oriented methods adopt asynchronous communication [26] which, however, introduces other problems: difficulty on verification of communication aspects and interference to inheritance of classes. New mechanisms need to be developed to accommodate both concurrency and inheritance coherently $[27,28,29,30]$. In addition to these concurrency related problems, the representation method for object-criented designs needs to be developed. A representation technique for object-oriented designs using a structured bipartite inheritance network has been developed [31], but representations for communication related aspects need to be introdued.

Partitioning and allocation of processes to hardware processors make the design of distributed software systems most distinct from the design of sequential software systems. Although substantial research has been done in this area $[32$, $33,34,35$ ], some practical problems, such as partitioning and allocation of tasks with precedence relations, still need to be solved. The problem of task partitioning is that we have to deal with two inherently conflicting criteria, namely min- 
imization of interprocess communication and maximization of parallelism. Another problem is that it is difficult to predict runtime behavior of the tasks before they are allocated. In measuring the effectiveness of task allocation, most of the cost functions are performance-oriented. Furthermore, since one of the advantages of distributed computing systems is its potential for fault tolerance, it is important to develop fault tolerant-oriented cost functions.

Design verification is one of the most important tasks in software design. In the design of distributed software systems, verification is more lifficult due to the complexity of such software systems. R:cently, methods for the verification of communication anc control flow of distributed software systems have been developed $[19,36,37]$. In [19], structured Petri net is used to analyze the control flow of distributed software systems. In [36], a formal method based on the inductive assertion method and Petri net for verification of synchronous communication is introduced. In [37] a formal method based on the context-free grammar is introduced to verify the control flow of distributed software systems. However, some important problems, such as verification of asynchronous communication and data flow in distributed software systems, remain unsolved. A formal methodology for the development of distributed software systems using attribute grammar and compiler techniques to represent the design specification and validate the implementation, has been proposed $[38,39]$. Nevertheless, more research related to formal lesign methodologies and the development of supporting tools for distributed software systems are still needed.

\section{References}

[1] D. R. Cheriton and W. Zwaenpoel, "Distributed Process Groups in the V Kernel," ACM Trans. on Computer Systems, Vol. 3, No. 2, 1985, pp. 77-107.

[2] R. F. Rashid, "Network Operating Systems," Local Area Networks: an Advanced Course, Lecture Notes in Computer Science, No. 184, Springer-Verlag, Berlin, West Germany, 1985, pp. 314-340.

[3] M. J. Weistein, T. W. Page, B. K. Livesey and G. J. Popek, "Transactions and Synchronization in a Distributed Operating System," Proc. 10th Symp. on Operating Systems Principles, 1985, pp. 115-125.

[4] Reference Manual for the Ada Programming Language, Ada Joint Program Office, Department of Defense, Washington, D. C., Oct. 1982.

[5] N. H., Gehani and W. D. Roome, The Concurrent C Programming Language, Silicon Press, Summit, N. J., 1989.

[6] Inmos Ltd., Occam Programming Language Manual, Prentice-Hall, Englew sod Cliffs, N. J., 1984.
[7] I. Sommerville, Software Engineering, 3rd cd., AddisonWesley Publishing Co., Wokingham, England, 1989.

[8] D. Marco, Structured Analysis and System Specification, Yourdon Press, New York, 1978.

[9] E. Yourdon and L. Constantine, Structured Design, 2nd ed., Yourdon Press, New York, 1978.

[10] E. Yourdon, Moder. Structured Analysis, Yourdon Press, New York, 1983.

[11] D. T. Ross, "Structured Analysis(SA): a language for communicating ideas," IEEE Trans. of Software Engineering, Vol. SE-3, No. 1, Jan. 1977, pp. 16-34.

[12] D. T. Ross, "Applications and Extensions of SADT," IEEE Computer, Vol. 18, No. 4, April 1985, pp. 25-34.

[13] P. T. Ward and S. J. Mellor, Structured Development for Real-Time Systems, Vols. 1, 2 and 3, Yourdon Press, New York, 1985.

[14] M. Alford, "SREM at the Age of Eight: The Distributed Computing Design System," IEEE Computer, Vol. 18, No. 4, April 1985, pp.36-45.

[15] D. Harel, H. Lachover, A. Naamad, A. Pnueli, M. Politi, R. Sherman, A. Shtull-Trauring and M. Trakhtenbrot, "STATEMATE: A Working Environment for the Development of Complex Reactive Systems," IEEE Trans. on Software Engineering, Vol. 16, No. 4, April, 1990, pp. 406-414.

[16] M. Evangelist, V. Y. Shen, I. R. Forman and M. Graf, "Using Raddle to Design Distributed Systems," Proc. 10th Int'l Conf. on Software Engineering, 1988, pp. 102111.

[17] S. S. Yau and M. U. Caglayan, "Distributed Software System Design Representation Using Modified Petri Nets," IEEE Trans, or Software Engineering, Vol. SE-9, No. 6, Nov. 1983 , pp. $733-745$.

[18] C. V. Ramamoorthy, Y. Yaw and W. T. Tsai, "Synthesis Rules for Cyclic Interaction among Processes in Concurrent Systems," Proc. 12th Annual Int'l Computer Software \& Applications Conf. (COMPSAC88), 1988, pp. $497-504$.

[19] S. S. Yau and C.-R Chou, "Control Flow Analysis of Distributed Computing System Software Using Structured Petri Net Model," Proc. Workshop on the Future Trends of Distributed Computing Systems in the 1990s, Sept. 1988, pp. 174-183.

[20] S. S. Yau and S. M. Shatz, "On Communication in the Design of Software Components of Distributed Computer Systems," Proc. Third Int'l Conf. on Distributed Computing Systems, Oct. 1982, pp.280-287. 
[21] G. Bruno and G. Marchetto, "Process-Translatable Petri Nets for the Rapid Prototyping of Process Control Systems," IEEE Trans. on Software Engineering, Vol. SE-12, No. 2, Feb. 1986, pp. 346-357.

[22] G. Bruno and A. Elia, "Operational Specification of Process Control Systems: Execution of PROT Nets Using OPS5," Proc. IFI' Congress'86, 1986, pp. 35-40.

[23] G. Booch, "Object-Oriented Development," IEEE Trans. on Software Ergineering, Vol. SE-12, No. 2, Feb. 1986, pp.211-221.

[24] I. Jackson, "Object Oriented Development in an Industrial Environment," Proc. Object-Oriented Programming Systems, Languages and Applications, ACM SIGPLAN Notices, Vol. 22, No. 12, 1987, pp. 183-191.

[25] S. S. Yau, C.-C. Yang and S. M. Shatz, "An Approach to Distributed Computing System Software Design," IEEE Trans. on Software Engineering, Vol. SE-7, No. 4, July 1981, pp. 427-436.

[26] G. Agha, Actors: A Model of Concurrent Computation in Distributed Systems, the MIT Press, MA., 1986.

[27] P. America, "Inheritance and Subtyping in a Parallel Object-Orientec Language," Proc. First European Conf. on Object-Oriented Programming, SpringerVerlag Lecture Notes in Computer Science 276, Berlin, West Germany, 1987, pp. 234-242.

[28] J.-P., Briot and A. Yonezawa, "Inheritance and Synchronization in Concurrent OOP," Proc. First European Conf. on Object-Oriented Programming, SpringerVerlag Lecture Notes in Computer Science 276, Berlin, West Germany, 1987, pp. 32-40.

[29] D. Decouchant, S. Krakowiak, M. Meysembourg, M. Riveill, and X. Rouscet de Pina, "A Synchronization Mechanism for Typed Objects in a Distributed System," Proc. ACM SIGPLAN Workshop on ObjectBased Concurrent Programming, SIGPLAN Notices, Vol. 24, No. 4, 1989, pp. 105-107.

[30] D. G. Kafura and K. H. Lee, "Inheritance in Actor Based Concurrent Object-Oriented Languages," Proc. Third European Conf. on Object-Oriented Programming, Stephen Cook (eds.), 1989, pp. 131-146.

[31] S. S. Yau and C.-S. Liu, "A Structured Inheritance Network Representation for Object-Oriented Software Design," Proc. 13th Annual Int'l Computer Software B' Applications Conf. (COMPSAC89), 1989, pp. 351-357.

[32] S. M. Shatz and S. S. Yau, "A Partitioning Algorithm for the Design of Distributed Software Systems," Infor. mation Sciences, Vol. 38, No. 2, April 1986, pp. 165-180.
[33] J. P. Huang, "Modeling of Software Partition for Distributed Real-Time Applications," IEEE Trans. on Software Engineering, Vol. 11, No. 10, Oct. 1985, pp.1113-1126.

[34] W. W. Chu and L. M.-T Lan, "Task Allocation and Precedence Relations for Distributed Real-Time Systems," IEEE Trans. on Computers, Vol. 36, No. 6, June 1987, pp. 667-679.

[35] V. M. Lo, "Heuristic Algorithms for Task Assignment in Distributed Systems," Proc. 4th Int'l Conf. on Distributed Computing Systems, 1984, pp. 30-39.

[36] S. S. Yau and K. W. Chen, "An Approach to Verification of Communication in Distributed Computing System Software," Proc. 9th Int'l Conf. on Distributed Computing Systems, June 1989, pp. 603-610.

[37] S. S. Yau and W. Hong, "Verification of Concurrent Control Flow in Distributed Computer Systems," IEEE Trans. on Software Engineering, Vol. 14, No. 4, April 1988, pp. 405-417.

[38] P. M. Lu, S. S. Yau and W. Hong, "A Formal Methodology Using Attributed Grammars for MultiprocessingSystem Software Development. I. Design Representation," Information Sciences, Vol. 30, 1983, pp. 79-105.

[39] P. M. Lu, S. S. Yau and W. Hong, "A Formal Methodology Using Attributed Grammars for MultiprocessingSystem Software Development. II. Validation," Information Sciences, Vol. 30, 1983, pp. 106-123. 